\title{
HIGH-RESOLUTION IMAGING OF BL LAC HOST GALAXIES
}

\author{
J. HEIDT \\ Landessternwarte, Königstuhl, 69117 Heidelberg, Germany \\ AND \\ T. PURSIMO, A. SILLANPÄ̈̈, L.O. TAKALO, K. NILSSON \\ Tuorla Observatory, Väisäläntie 20, 21500 Piikkiö, Finland
}

We present high-resolution imaging of the BL Lac objects 1ES $0229+200(\mathrm{z}$ $=0.14)$, Markarian $421(\mathrm{z}=0.031)$ and the prototype BL Lac $(\mathrm{z}=0.069)$. Our goal is to study the properties of the host galaxies of this enigmatic sources and to compare them with their non-actice counterparts. The observations were carried out with the Nordical Optical Telescope (NOT) at La Palma under sub-arcsecond seeing conditions (FWHM <0." 8 ).

The surface brightness profiles of the host galaxies were analyzed as described in Bender and Möllenhoff ( $A \& A, \mathbf{1 7 7}, 71$ (1987)). After masking the disturbing field stars/galaxies isophotes were fitted by ellipses down to $20.5 \mathrm{mag} / \mathrm{arcsec}^{2}$ in Markarian 421, $23.5 \mathrm{mag} / \mathrm{arcsec}^{2}$ in BL Lac and $25.5 \mathrm{mag} / \mathrm{arcsec}^{2}$ in 1ES 0229+200. Additionally, we analyzed the surface brightness profile of the companion of Markarian 421.

All BL Lac objects are embedded in giant elliptical galaxies. Their host galaxies show ellipticities in the range from 0.2 to 0.4 and constant position angles. The $\alpha_{4}$-Fourier coefficient is consistent with zero in 1ES $0229+200$ and Markarian 421 and is positive in BL Lac. This is already the third BL Lac object, where "disky" isophotes have been found in their host galaxies. The companion of Markarian 421 shows a strong bulge and very high positive $\alpha_{4}$-Fourier coefficients. Hence it is likely that this galaxy is an early-type spiral, contradictionary to previous claims in the literature.

In order to estimate an absolute magnitude for the host galaxies of the three BL Lac objects we fitted a de Vaucouleurs profile to the observed surface brightness profile excluding the inner $3 \times \mathrm{FWHM}$ arcsec from the fit. We derived $M_{R}=-24.1$ for $1 \mathrm{ES} 0229+200, M_{R}=-25.1$ for Markarian 421 and $M_{R}=-24.5$ for BL Lac $\left(H_{0}=50 \mathrm{~km} / \mathrm{s} / \mathrm{Mpc}, q_{0}=0\right)$. They are in the same range as determined in other studies. 